\title{
Effective spin-1 Heisenberg chain in coupled cavities
}

\author{
Ling Zhou, Wei-Bin Yan, Xin-Yu Zhao
}

\begin{abstract}
A coupled array of $N$ identical cavities, each of which contains a five-level atom is investigated. The results show that the atoms via the exchange of virtual photons can be effectively equal to spin 1 Heisenberg model under certain conditions. By tuning the laser fields, the parameters of the effective Hamiltonian can be controlled individually.
\end{abstract}

PACS numbers: 03.67.Mn, 42.50.Dv, 75.10.Jm

\section{INTRODUCTION}

Spin chain has been played an important role in quantum information field as well as in condensed matter physics. The interaction between on site spin can offer us entanglement in solid and realistic way [1-7]. It has been found that spin chain with an open boundary condition can be applied in quantum communication [8], which can translate information with high fidelity from one end to another. Perfect state transfer can be realized by changing the interaction between the qubits in spin networks [9, 10].

However, because of the microscopic properties of solid-state materials, it is very hard to address individual spins while it is the prerequisite for quantum information processing. Single spin addressability can also be very helpful to obtain deeper and more detail insight into condensed matter physics. In order to do this, it has been show that the arrays of Josephson junctions [11, quantum dots [12], optical lattices 13], can provide effective spin-chain Hamiltonian where spin-coupling constants can be controlled. Recently under active investigation is to use the array of coupled cavities, which are ideally suited to addressing individual spins [14-23]. Intense interest has arisen from the demonstration that a polaritonic Mott transition and a Bose-Hubbard interaction can be generated in these structures [15, 16, 17]. Hartmann [18] have shown that single atoms in interacting cavities that are operated in a strong coupling regime can form a Heisenberg spin $\frac{1}{2}$ Hamiltonian in which all parameters of the effective Hamiltonian can be tuned individually.

However, people are increasedly interested in the multilevel systems. A lots of papers treat with multilevel systems in different areas of physics such as in condensed matter physics [19, 20], statistical mechanics [21, 22], as well as in quantum information [23, 24, 25]. Now multilevel systems can be considered as a important field. In this paper, we consider a coupled array of $N$ identical cavities, each of which contain a five-level atom. We show that under large detuning case the atoms via the exchange of virtual photons can be effectively equal to spin 1 model. We use atomic bare basis as spin-level. It should be easy to manipulate the individual atom when we need to project measure etc. on the individual qutrit.

\section{MODEL AND THE EFFECTIVE}

\section{HAMILTONIAN}

We consider an array of cavities which are coupled via exchange of photons. Each of the cavities contains one five-level atom. The atomic configuration is shown in Fig.1. The three long-lived levels $|a\rangle,|b\rangle,|c\rangle$ represent the three spin states. The cavity mode couples to the transitions $|d\rangle \leftrightarrow|a\rangle,|d\rangle \leftrightarrow|b\rangle,|e\rangle \leftrightarrow|b\rangle$, and $|e\rangle \leftrightarrow|c\rangle$ while four lasers drive the atom with Rabi frequencies $\Omega_{i}(i=1,2,3,4)$, respectively. The same atomic configurations has been used in 30] for addressing individual atoms in optical lattices with standing-wave driving fields. In the interaction picture, the Hamiltonian reads

$$
\begin{aligned}
H= & \sum_{j=1}^{N}\left[\left(g_{1} a_{j}\left|d_{j}\right\rangle\left\langle a_{j}\left|+\Omega_{1}\right| d_{j}\right\rangle\left\langle b_{j}\right|\right) e^{i \Delta_{1} t}\right. \\
& +\left(g_{2} a_{j}\left|d_{j}\right\rangle\left\langle b_{j}\left|+\Omega_{2}\right| d_{j}\right\rangle\langle a|\right) e^{i \Delta_{2} t} \\
& +\left(g_{3} a_{j}\left|e_{j}\right\rangle\left\langle b_{j}\left|+\Omega_{3}\right| e_{j}\right\rangle\left\langle c_{j}\right|\right) e^{i \Delta_{3} t} \\
& \left.+\left(g_{4} a_{j}\left|e_{j}\right\rangle\left\langle c_{j}\left|+\Omega_{4}\right| e_{j}\right\rangle\left\langle b_{j}\right|\right) e^{i \Delta_{4} t}+h . c .\right] \\
& +\sum_{j=1}^{N} J\left(a_{j}^{\dagger} a_{j+1}+a_{j} a_{j+1}^{\dagger}\right)
\end{aligned}
$$

where $g_{i}$ is the coupling strength between the cavity and the atom, and $\Delta_{i}$ express the detuning shown in Fig.1, and $a_{j}$ means the annihilation operator of the cavity field. Here, for simplicity, we assume that the coupling strength $g_{i}$ and laser Rabi frequency $\Omega_{i}$ do not change with cavity sequence $j$, which means that the identical atoms are in the identical cavities and driven by identical laser fields. The first sum denote the interaction within the cavities, and the second sum is the intercavity hopping between nearest neighbor with hopping rate $J$. Now we consider the large detuning case with $\left|\Delta_{i}\right| \gg\left|g_{i}\right|,\left|\Omega_{i}\right|$. In addition, in order to avoid undesired atomic transitions, we need the following inequalities $\Delta_{2}-\Delta_{1} \gg\left\{\frac{\Omega_{1} \Omega_{2}}{\Delta_{1}}, \frac{\Omega_{1} g_{2}}{\Delta_{1}}, \frac{g_{1} \Omega_{2}}{\Delta_{1}}, \frac{g_{1} g_{2}}{\Delta_{1}}\right\}, \Delta_{4}-\Delta_{3} \gg$ $\left\{\frac{\Omega_{3} \Omega_{4}}{\Delta_{3}}, \frac{\Omega_{3} g_{4}}{\Delta_{3}} \frac{g_{3} \Omega_{4}}{\Delta_{3}} \frac{g_{3} g_{4}}{\Delta_{3}}\right\}$. We adiabatic eliminate atomic excited state $|d\rangle$ and $|e\rangle$ and have 


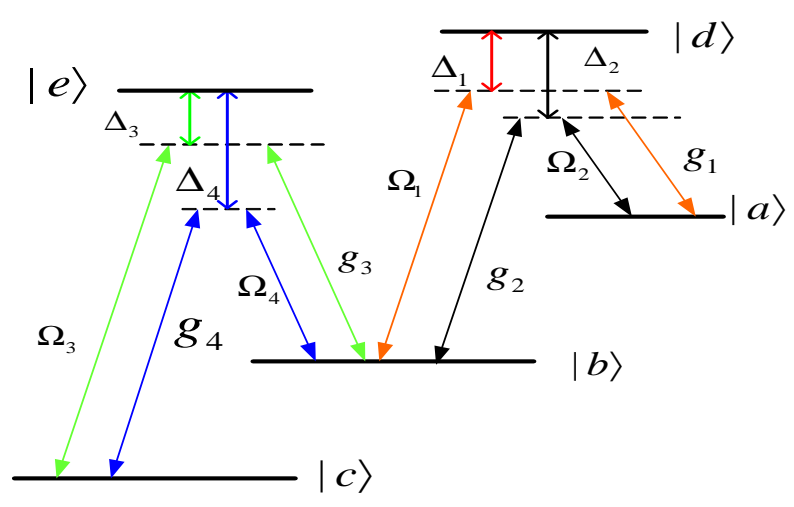

FIG. 1: (Color online) The configuration of the five-level atom. The three long-lived levels $|a\rangle,|b\rangle,|c\rangle$ represent the three spin states. The cavity mode couples to the transtions $|d\rangle \leftrightarrow|a\rangle,|d\rangle \leftrightarrow|b\rangle,|e\rangle \leftrightarrow|b\rangle$, and $|e\rangle \leftrightarrow|c\rangle$ while four lasers drive the atom with Rabi frequencies $\Omega_{i}(i=1,2,3,4)$, respectively.
Noting that the every term in $H_{0}$ commutes each other, when we perform unitary transformation we can do it separately. Diagonalize the cavity-hopping terms by employing a Fourier transformed basis as $a_{j}=\frac{1}{\sqrt{N}} \sum_{k=1}^{N} F_{j k} b_{k}$, where $F_{j k}=\exp \left(-i \frac{2 \pi}{N} j k\right)$ and $\sum_{j=1}^{N} F_{j k} F_{j l}^{*}=N \delta_{k l}$. The diagonalized form reads $J \sum_{j}\left(a_{j}^{\dagger} a_{j+1}+a_{j} a_{j+1}^{\dagger}\right)=\sum_{j} \nu_{j} b_{j}^{\dagger} b_{j}$ with $\nu_{j}=$ $2 J \cos \left(\frac{2 \pi}{N} j\right)$. Now, we goes into the new frame rotating with $H_{0}$, under the conditions

$$
\begin{aligned}
\frac{\Omega_{1}^{2}}{\Delta_{1}}+\frac{\Omega_{4}^{2}}{\Delta_{4}} & =\frac{1}{2}\left(\frac{\Omega_{2}^{2}}{\Delta_{2}}+\frac{\Omega_{3}^{2}}{\Delta_{3}}\right), \\
\frac{\Omega_{1} g_{1}}{\Delta_{1}} & =\frac{\Omega_{3} g_{3}}{\Delta_{3}}, \frac{\Omega_{2} g_{2}}{\Delta_{2}}=\frac{\Omega_{4} g_{4}}{\Delta_{4}},
\end{aligned}
$$

we have the Hamiltonian

$$
\begin{aligned}
H= & -\sum_{j=1}^{N}\left\{\left[\frac{\Omega_{1} g_{1}}{\Delta_{1}}\left(\left|b_{j}\right\rangle\left\langle a_{j}|+| c_{j}\right\rangle\left\langle b_{j}\right|\right) e^{i \mu_{+} t}\right.\right. \\
& \left.+\frac{\Omega_{2} g_{2}}{\Delta_{2}}\left(\left|a_{j}\right\rangle\left\langle b_{j}|+| b_{j}\right\rangle\left\langle c_{j}\right|\right) e^{i \mu_{-} t}\right] \\
& \left.e^{-i \nu_{j} t} F_{j k} b_{k}+\text { h.c. }\right\}
\end{aligned}
$$

$$
\begin{aligned}
& -\sum_{j=1}^{N}\left[\frac{\Omega_{2}^{2}}{\Delta_{2}}\left|a_{j}\right\rangle\left\langle a_{j}\left|+\left(\frac{\Omega_{1}^{2}}{\Delta_{1}}+\frac{\Omega_{4}^{2}}{\Delta_{4}}\right)\right| b_{j}\right\rangle\left\langle b_{j}\left|+\frac{\Omega_{3}^{2}}{\Delta_{3}}\right| c_{j}\right\rangle\left\langle c_{j}\right|\right] \\
& -\sum_{j=1}^{N}\left[\frac{\Omega_{1} g_{1}}{\Delta_{1}} a_{j}\left|b_{j}\right\rangle\left\langle a_{j}\left|+\frac{\Omega_{2} g_{2}}{\Delta_{2}} a_{j}\right| a_{j}\right\rangle\left\langle b_{j}\right|\right. \\
& \left.+\frac{\Omega_{3} g_{3}}{\Delta_{3}} a_{j}\left|c_{j}\right\rangle\left\langle b_{j}\left|+\frac{\Omega_{4} g_{4}}{\Delta_{4}} a_{j}\right| b_{j}\right\rangle\left\langle c_{j}\right|+h . c .\right] \\
& +\sum_{j=1}^{N} J\left(a_{j}^{\dagger} a_{j+1}+a_{j} a_{j+1}^{\dagger}\right) .
\end{aligned}
$$$$
H=-\sum_{j=1}^{N}\left[\frac{g_{1}^{2}}{\Delta_{1}}\left|a_{j}\right\rangle\left\langle a_{j}\left|+\left(\frac{g_{2}^{2}}{\Delta_{2}}+\frac{g_{3}^{2}}{\Delta_{3}}\right)\right| b_{j}\right\rangle\left\langle b_{j}\left|+\frac{g_{4}^{2}}{\Delta_{4}}\right| c_{j}\right\rangle\left\langle c_{j}\right|\right] a_{j}^{\dagger} a_{j}
$$

with $\mu_{ \pm}=\frac{g_{1}^{2}}{\Delta_{1}} \pm \frac{1}{2}\left(\frac{\Omega_{2}^{2}}{\Delta_{2}}-\frac{\Omega_{3}^{2}}{\Delta_{3}}\right)$. We define that the eigenstates of $S_{z}$ for $S=1$ are atomic bare level $|a\rangle,|b\rangle$, $|c\rangle$; therefore $S_{i+}=S_{i x}+i S_{i y}=\sqrt{2}(|a\rangle\langle b|+| b\rangle\langle c|)$, $S_{i-}=S_{i x}-i S_{i y}=\sqrt{2}(|b\rangle\langle a|+| c\rangle\langle b|)$. Using the spin operator, we rewrite the Hamiltonian Eq.(7) as

$$
\begin{aligned}
H= & -\sum_{j, k=1}^{N}\left[\left(\frac{\Omega_{1} g_{1}}{\sqrt{2} \Delta_{1}} S_{j}^{-} e^{i \mu_{+} t}\right.\right. \\
& \left.\left.+\frac{\Omega_{2} g_{2}}{\sqrt{2} \Delta_{2}} S_{j}^{+} e^{i \mu_{-} t}\right) e^{-i \nu_{k} t} F_{j k} b_{k}+\text { h.c. }\right] .
\end{aligned}
$$

We assume

$$
\frac{g_{1}^{2}}{\Delta_{1}}=\frac{g_{2}^{2}}{\Delta_{2}}+\frac{g_{3}^{2}}{\Delta_{3}}=\frac{g_{4}^{2}}{\Delta_{4}}
$$

The effective Hamiltonian can be classified as

$$
H=H_{0}+H_{1}
$$

with

$$
\begin{aligned}
H_{0}= & -\sum_{j=1}^{N}\left[\frac{g_{1}^{2}}{\Delta_{1}} a_{j}^{\dagger} a_{j}-J\left(a_{j}^{\dagger} a_{j+1}+a_{j} a_{j+1}^{\dagger}\right)\right] \\
& -\sum_{j=1}^{N}\left[\frac{\Omega_{2}^{2}}{\Delta_{2}}\left|a_{j}\right\rangle\left\langle a_{j}\left|+\left(\frac{\Omega_{1}^{2}}{\Delta_{1}}+\frac{\Omega_{4}^{2}}{\Delta_{4}}\right)\right| b_{j}\right\rangle\left\langle b_{j}\left|+\frac{\Omega_{3}^{2}}{\Delta_{3}}\right| c_{j}\right\rangle\left\langle c_{j}\right|\right], \\
H_{1}= & -\sum_{j=1}^{N}\left[\frac{\Omega_{1} g_{1}}{\Delta_{1}} a_{j}\left|b_{j}\right\rangle\left\langle a_{j}\left|+\frac{\Omega_{2} g_{2}}{\Delta_{2}} a_{j}\right| a_{j}\right\rangle\left\langle b_{j}\right|\right. \\
& \left.+\frac{\Omega_{3} g_{3}}{\Delta_{3}} a_{j}\left|c_{j}\right\rangle\left\langle b_{j}\left|+\frac{\Omega_{4} g_{4}}{\Delta_{4}} a_{j}\right| b_{j}\right\rangle\left\langle c_{j}\right|+\text { h.c. }\right] .
\end{aligned}
$$

If the Rabi frequency $\left\{\frac{\Omega_{1} g_{1}}{\sqrt{2} \Delta_{1}}, \frac{\Omega_{2} g_{2}}{\sqrt{2} \Delta_{2}}\right\} \ll\left\{\left|\mu_{+}-\nu_{k}\right|, \mid \mu_{-}-\right.$ $\left.\nu_{k} \mid\right\}$, this allows us to make use of the adiabatic elimination once more. Considering the subspace without real photons, we deduce the effective Hamiltonian as

$H=\sum_{j=1}^{N}\left[\frac{\Omega_{1}^{2} g_{1}^{2}}{2 \Delta_{1}^{2}} \frac{1}{\mu_{+}-\nu_{k}} S_{j}^{-} S_{l}^{+}+\frac{\Omega_{2}^{2} g_{2}^{2}}{2 \Delta_{2}^{2}} \frac{1}{\mu_{-}-\nu_{k}} S_{j}^{+} S_{l}^{-}\right] F_{j k} F_{l k}^{*}$.

In view of $\nu_{k}=J\left(F_{1 k}+F_{1 k}^{*}\right)$, we expand $\frac{F_{j k} F_{l k}^{*}}{\mu_{ \pm}-\nu_{k}}$ as

$$
\frac{F_{j k} F_{l k}^{*}}{\mu_{ \pm}-\nu_{k}}=\frac{1}{\mu_{ \pm}}\left(F_{j k} F_{l k}^{*}+\frac{J}{\mu_{ \pm}} F_{j+1 k} F_{l k}^{*}+\frac{J}{\mu_{ \pm}} F_{j k} F_{l+1 k}^{*}\right) .
$$

This expand demand the condition $J \prec \mu_{ \pm}$. Substitute the relation Eq.(10) into Eq.(9), we finally obtain the effective Hamiltonian

$$
H_{x y}=\sum_{j=1}^{N} A\left[S_{x j}^{2}+S_{y j}^{2}\right]+B S_{j z}+C\left(S_{x j} S_{x j+1}+S_{y j} S_{y j+1}\right)
$$



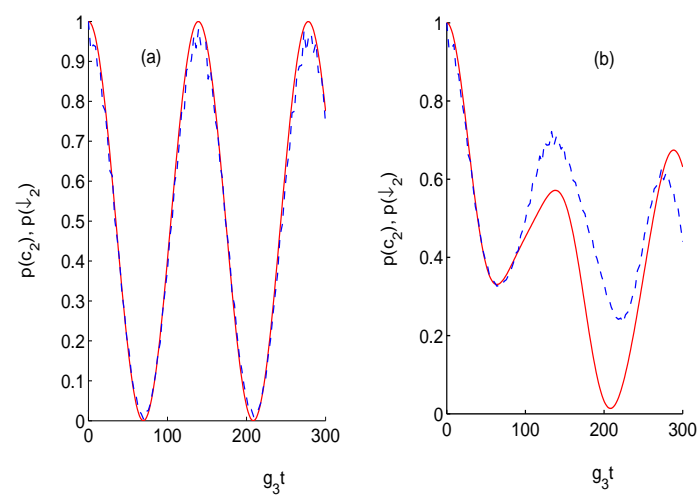

FIG. 2: (Color online) The comparison of the probility $p\left(c_{2}\right)$ [ $\left.p\left(\downarrow_{2}\right)\right]$ between the Hamiltonian Eq. (2)(blue-dashed line) with the effective spin chain Eq.(11)(red-solid line). (a): the initial atomic state is $\left|b_{1}, c_{2}\right\rangle$. (b): the initial atomic state is $\frac{1}{2}\left(\left|a_{1}\right\rangle\left\langle a_{1}|+| b_{1}\right\rangle\left\langle b_{1}\right|\right) \otimes\left|c_{2}\right\rangle\left\langle c_{2}\right|\left(\frac{1}{2}\left(\left|\uparrow_{1}\right\rangle\left\langle\uparrow_{1}|+| \rightarrow_{1}\right\rangle\left\langle\rightarrow_{1}\right|\right) \otimes \mid \downarrow_{2}\right.$ \rangle$\left\langle\downarrow_{2}\right|$ for spin chain Eq.(11). For all plots, the parameters are $g_{1}=g_{2}=g_{4}=1, g_{3}=1 / 2, \Delta_{1}=\Delta_{4}=40, \Delta_{3}=20$, $\Delta_{2}=80, \Omega_{1}=\Omega_{2}=\Omega_{3}=10, \Omega_{4}=5, J=0.5$.

with

$$
\begin{aligned}
A & =\frac{\Omega_{1}^{2} g_{1}^{2}}{2 \Delta_{1}^{2} \mu_{+}}+\frac{\Omega_{2}^{2} g_{2}^{2}}{2 \Delta_{2}^{2} \mu_{-}}, B=\frac{\Omega_{2}^{2} g_{2}^{2}}{2 \Delta_{2}^{2} \mu_{-}}-\frac{\Omega_{1}^{2} g_{1}^{2}}{2 \Delta_{1}^{2} \mu_{+}}, \\
C & =\frac{\Omega_{1}^{2} g_{1}^{2} J}{\Delta_{1}^{2} \mu_{+}^{2}}+\frac{\Omega_{2}^{2} g_{2}^{2} J}{\Delta_{2}^{2} \mu_{-}^{2}} .
\end{aligned}
$$

We see clearly that it is spin $1 \mathrm{XY}$ antiferromagnetic Hamiltonian $(C>0)$. Because it is spin 1, the term $S_{x j}^{2}+S_{y j}^{2}$ can not be omitted, which is of essential importance in high-spin cases. Although individual control of the coefficients $A, B, C$ is limited owing to their mutual dependency, we still can change them; because $\left\{g_{i}, \Delta_{i}, \Omega_{i}\right\}(i=1 \ldots 4)$ meeting with the condition Eqs.(3),(6), we still have seven variable so as to adjust the coefficients $A, B, C$.

To confirm the validity of our approximations, we numerically simulate the dynamics generated by Hamiltonian Eq.(2) and compare it to the dynamics generated by effective spin model Eq.(11). In Fig. 2a, we consider two atoms in two cavities initially in the state $\left|b_{1}, c_{2}\right\rangle$ (atom one in $|b\rangle$ and atom two in $|c\rangle$ ) and calculate the occupation probability $p\left(c_{2}\right)$ (atom 2 in state $|c\rangle)$ which corresponding to the probability of spin 2 to point down. Fig.2a shows the time evolution of $p\left(c_{2}\right)$ for Hamiltonian Eq.(2) and $p\left(\downarrow_{2}\right)$ for effective spin mode Eq.(11). For our choice of the parameters, one can easy check that they satisfy all kinds of approximation condition such as Eqs.(3), (6) and the two times adiabatic elimination condition $\left|\Delta_{i}\right| \gg\left\{\left|g_{i}\right|,\left|\Omega_{i}\right|\right\}, \Delta_{2}-\Delta_{1} \gg\left\{\frac{\Omega_{1} \Omega_{2}}{\Delta_{1}}, \frac{\Omega_{1} g_{2}}{\Delta_{1}}, \frac{g_{1} \Omega_{2}}{\Delta_{1}}, \frac{g_{1} g_{2}}{\Delta_{1}}\right\}$, $\Delta_{4}-\Delta_{3} \gg\left\{\frac{\Omega_{3} \Omega_{4}}{\Delta_{3}}, \frac{\Omega_{3} g_{4}}{\Delta_{3}} \frac{g_{3} \Omega_{4}}{\Delta_{3}} \frac{g_{3} g_{4}}{\Delta_{3}}\right\}$, and $\left\{\frac{\Omega_{1} g_{1}}{\sqrt{2} \Delta_{1}}, \frac{\Omega_{2} g_{2}}{\sqrt{2} \Delta_{2}}\right\} \ll\left\{\left|\mu_{+}-\nu_{k}\right|,\left|\mu_{-}-\nu_{k}\right|\right\}$. From the Fig. 2a, we know that the interaction can be effectively equal to spin 1 Heisenberg chain because the two

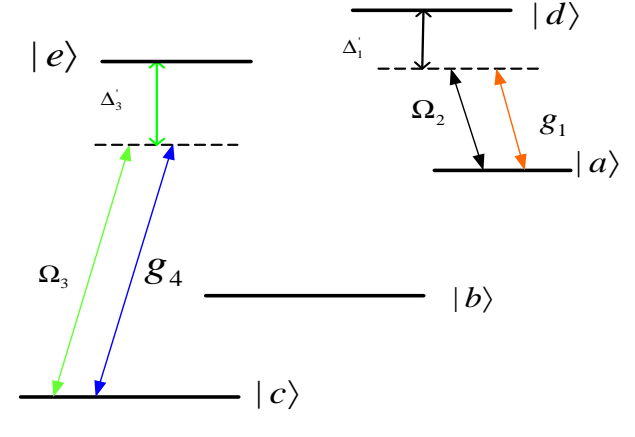

FIG. 3: (Color online) The illustration to get $S_{Z} S_{Z}$ interaction. Two classical fields are deleted

curves almost merge into one. For the initial atomic state $\left|b_{1}, c_{2}\right\rangle$, the population of two atoms just oscillate between the two level, and level $|a\rangle$ do not participate the interaction so that we have the reduced two-level system (level $|a\rangle$ has no population).

Fig.2b plots the population $p\left(c_{2}\right)$ for initial state $\frac{1}{2}\left(\left|a_{1}\right\rangle\left\langle a_{1}|+| b_{1}\right\rangle\left\langle b_{1}\right|\right) \otimes\left|c_{2}\right\rangle$. Under this case, all the three levels participate the interaction so that we can see the two steps oscillation (see red line). But now, we have much discrepancy between Hamiltonian Eq. (2) and effective Hamiltonian Eq. (11). The discrepancies come out of the higher order term in the adiabatic elimination. Due to the two participators the excited level and the middle level, the discrepancy between Hamiltonian Eq. (2) and effective Hamiltonian Eq. (11) become larger.

Now, we will obtain effective $S_{Z} S_{Z}$ interaction. We still employ the atomic level configuration but now we only need the two laser beams working between $|e\rangle \leftrightarrow|c\rangle$ and $|d\rangle \leftrightarrow|a\rangle$ shown in Fig.3. The Hamiltonian is as

$$
\begin{aligned}
H= & \sum_{j=1}^{N}\left[\left(g_{1} a_{j}+\Omega_{2}\right)\left|d_{j}\right\rangle\left\langle a_{j}\right| e^{i \Delta_{1}^{\prime} t}+\left(g_{4} a_{j}+\Omega_{3}\right)\right. \\
& \left.\left.\left|e_{j}\right\rangle\left\langle c_{j}\right|\right) e^{i \Delta_{3}^{\prime} t}+\text { h.c. }\right]+\sum_{j=1}^{N} J\left(a_{j}^{\dagger} a_{j+1}+a_{j} a_{j+}^{\dagger}(1) 3\right.
\end{aligned}
$$

The other two cavity fields denoting with $g_{2}$ and $g_{3}$ in

Eq. (1) will only induce a Stark shift on the level $|b\rangle$ and do not affect the effective interaction, so we do not write it in Eq. (13). Under larger detuning $\left\{\left|\Delta_{1}^{\prime}\right|,\left|\Delta_{3}^{\prime}\right|\right\} \gg$ $\left\{g_{1}, g_{4}, \Omega_{2} \Omega_{3}, J\right\}$, we adiabatic eliminate atomic excited 
state and have

$$
\begin{aligned}
H= & -\sum_{j=1}^{N}\left\{\frac{g_{1}^{2}}{\Delta_{1}^{\prime}}\left[\left|a_{j}\right\rangle\left\langle a_{j}|+| c_{j}\right\rangle\left\langle c_{j}\right|\right] a_{j}^{\dagger} a_{j}-\nu_{j} b_{j}^{\dagger} b_{j}\right\} \\
& -\sum_{j=1}^{N}\left[\frac{\Omega_{3}^{2}}{\Delta_{3}^{\prime}}\left|c_{j}\right\rangle\left\langle c_{j}\left|+\frac{\Omega_{2}^{2}}{\Delta_{1}^{\prime}}\right| a_{j}\right\rangle\left\langle a_{j}\right|\right] \\
& -\sum_{j=1}^{N}\left[\frac{\Omega_{2} g_{1}}{\Delta_{1}^{\prime}}\left(a_{j} S_{z j}+\text { h.c. }\right)\right.
\end{aligned}
$$

where we need the condition $\frac{g_{1}^{2}}{\Delta_{1}^{\prime}}=\frac{g_{4}^{2}}{\Delta_{3}^{\prime}}, \frac{\Omega_{3} g_{4}}{\Delta_{3}^{\prime}}=-\frac{\Omega_{2} g_{1}}{\Delta_{1}^{\prime}}$. Switch into interaction picture and then adiabatic eliminate once more under the condition $\left|\frac{\Omega_{2} g_{1}}{\Delta_{1}^{\prime}}\right| \ll\left|\frac{g_{1}^{2}}{\Delta_{1}^{\prime}}-\nu_{k}\right|$. Finally, we have the effective Hamiltonian

$$
H_{z z}=\sum_{j=1}^{N} \frac{\Omega_{2}^{2} g_{1}^{2}}{\Delta_{1}^{\prime 2} u} S_{z j}^{2}+\frac{2 J \Omega_{2}^{2} g_{1}^{2}}{\Delta_{1}^{\prime 2} u^{2}} S_{z j} S_{z j+1} .
$$

where $u=\frac{g_{1}^{2}}{\Delta_{1}^{\prime}}$. Now, the effective Hamiltonian is $S_{z} S_{z}$ interaction.

During the process of deduction of effective Hamiltonian Eqs.(11) and (15), we have changed working picture for two times. But the atomic population probability do not change with the changing of the picture. So, we can compare the atomic population probability in different picture, for example we have done in Fig. 2. However, the Hamiltonian Eqs. (13) and (15) do not affect atomic population probability. Therefore, we do not plot it again.

The two Hamiltonians Eqs. (11) and (15) can be combined into one effective Hamiltonian if we employ the method proposed by [18]. The lasers that generate the
Hamiltonian (11) are turned on for a short time interval $d t\left(\left\|H_{x y}\right\| d t \ll 1\right)$ followed by another time interval $d t$ $\left(\left\|H_{z z}\right\| d t \ll 1\right)$ with the lasers that generate the Hamiltonian (15) turned on. By repeating this sequence until the total time range to be simulated is covered. The effective Hamiltonian $H=H_{x y}+H_{z z}$ finally can be obtained.

The decoherence of the system mainly results from the decay mechanisms via the photons or the excited state $|e\rangle(|d\rangle)$. To overcome the decoherence, the coefficients of effective Hamiltonian $A, B$, and $C$ should be much larger than the decays rates of photons and the excited state $|e\rangle$ $(|d\rangle)$. Using the group of the parameters of Fig. 2 (all the parameters are scaled in $g_{1}$ ), we have $A=-0.0128$, $B=0.0210, C=0.0113$. For a strongly coupled single quantum dot-cavity system, $\frac{g}{\kappa} \sim 1800(\kappa \sim 1 / 1800)$, $\frac{g}{\gamma} \sim 300(\gamma \sim 1 / 300)$ in which $\kappa(\gamma)$ means the decay of the cavity ( the excited state ) have been achieved for off resonance [32]. Therefore $\{A, B, C\} \ll\{\kappa, \gamma\}$ can be realized in experiment.

\section{CONCLUSION}

We consider a coupled array of $N$ identical cavities, each of which contains a five-level atom. We show that under large detuning case the atoms via the exchange of virtual photons can be effectively equal to spin 1 Heisenberg model. Although the coefficients are related one another, we still can tune them by controlling the laser fields so that our system is a good simulator for spin 1 Heisenberg model. When operated in a two-dimensional array of cavities the device is thus able to simulate spin lattices.

Acknowledgments: The project was supported by NSFC under Grant No.10774020, and also supported by SRF for ROCS, SEM.
[1] Arnesen M C, Bose S and Vedral V 2001 Phys. Rev. Lett. 87017901

[2] Wang X 2001 Phys. Rev. A 64012313

[3] Kamta G L and Starace A F 2002 Phys. Rev. Lett. 88 107901

[4] Zhou L, Song H S, Guo Y Q, and Li C 2003 Phys. Rev. A 68024301

[5] Zhang G F, Li S S 2005 Phys. Rev. A 72034302

[6] Abliz A, Gao H J, Xie X C, Wu Y S, and Liu W M 2006 Phys. Rev. A 74052105

[7] Kheirandish F, Akhtarshenas S J, Mohammadi H 2008 Phys. Rev. A 77042309

[8] Bose S 2003 Phys. Rev. Lett. 91207901

[9] Christandl M, Datta N, Ekert A, and Landahl A J 2004 Phys. Rev. Lett. 92187902

[10] Wojcik A, Luczak T, Kurzynski P, Grudka A, Gdala T, and Bednarska M 2005 Phys. Rev. A 72034303

[11] Bruder C, Fazio R, and Schön G 1993 Phys. Rev. B 47 342

[12] Loss D and DiVincenzo D P 1998 Phys. Rev. A 57 120;
Burkard G, Loss D and DiVincenzo D P 1999 Phys. Rev. B 592070

[13] Duan L-M, Demler E, and Lukin M D 2003 Phys. Rev. Lett. 91 090402; Garcia-Ripoll J J, Martin-Delgado M A, and Cirac J I 2004 Phys. Rev. Lett. 93250405

[14] Kane B E 1998 Nature 393133

[15] Angelakis D G, Santos M F, and Bose S 2007 Phys. Rev. A $76031805(\mathrm{R})$

[16] Hartmann M J, Brandão F G S L, and Plenio M B 2006 Nature Phys. 2849

[17] Greentree A D, Tahan C, Cole J H, and Hollenberg L C L 2006 Nature Phys. 2856

[18] Hartmann M J, Brandão F G S L, and Plenio M B 2007 Phys. Rev. Lett. 99160501

[19] Nemoto K, Holmes C A, Milburn G J, and Munro W J 2001 Phy. Rev. A 63013604

[20] Foerster A, Ragoucy E 2007 Nuclear Phys. B 777373

[21] Yip S K 2003 Phys. Rev. Lett. 90250402

[22] Pires A S T, Costa B V, and Dias R A 2008 Phys. Rev. B 78212408 
[23] Romero-Isart O, Eckert K, and Sanpera A 2007 Phys. Rev. A 75050303

[24] Alipour S, Karimipour V, and Memarzadeh L 2007 Phys. Rev. A 75 052322;Zhou L, Yi Y X, Song H S 2005 Chin. Phys. 141168

[25] Bruss D and Macchiavello C 2002 Phys. Rev. Lett. 88 127901; Kaszlikowski D, Gnaciński P, Żukowski M, Miklaszewski W, and Zeilinger A 2000 ibid. 854418

[26] Rossini D and Fazio R 2007 Phys. Rev. Lett. 99186401

[27] Paternostro M, Agarwal G S, and Kim M
S arXiv:0707.0846

[28] Cho J., Angelakis D. G., and Bose S., arXiv:0712.2413

[29] Li Y., Huo M X, Song Z, and Sun C P, arXiv:0802.0079. Hu F M, Zhou Lan, Shi Tao, and Sun C P 2007 Phys. Rev. A 76013819

[30] Cho J 2007 Phys. Rev. Lett. 99020502

[31] Kay A and Angelakis D G 2008 EPL 8420001

[32] Hennessy K et al. 2007 Nature (London) 445896 